\title{
Predicting the target genes of miRNAs in preterm via targetscore algorithm
}

\author{
KEXIN LU ${ }^{1}$, JUNZHI HUANG ${ }^{1}$, YANDONG YANG ${ }^{1}$ and DONGLI LU ${ }^{2}$ \\ Departments of ${ }^{1}$ Obstetrics and Gynecology and ${ }^{2}$ Neurology, \\ Binzhou Medical University Hospital, Binzhou, Shandong 256603, P.R. China
}

Received May 28, 2018; Accepted December 7, 2018

DOI: $10.3892 /$ etm.2019.7179

\begin{abstract}
Compared with normal neonates, preterm infants have an immature immune system which causes them to have a higher morbidity rate and even death. In order to reduce the mortality of newborns, we need to find the target genes which affect the preterm and understand their mechanism. It has been verified that microRNA (miRNA)-200 and miRNA-182 are closely related to the incidence of preterm. Therefore, it is significant to predict the target genes which are regulated by them for further understanding the mechanism of preterm. We chose the targetscore method for calculating the variational Bayesian-Gaussian mixture model (VB-GMM) as the target genes prediction method. It is designed for condition-specific target predictions and not limited to predict conserved genes, so the results are more accurate than previous sequence-based target prediction algorithms. In this study, our major contribution is to predict the target mRNAs of the chosen miRNAs with the gene expression profiles and a new method, which can effectively improve the accuracy of the prediction.
\end{abstract}

\section{Introduction}

Preterm is a situation in which the pregnant women have a delivery during pregnancy between 28-37 weeks. Compared with normal infants, preterm infants have an immature immune system which lead to higher morbidity (1). Therefore, preterm is a significant cause for the death of perinatal infants (2). However, present research is still not able to explain clearly the mechanism of the occurrence of preterm, including the theory of cervical ripening, neurotransmitter (3), immune, mechanical hypothesis (4) and endocrine control (5). Due to the lack of the detailed molecular characterizations and understanding of gene-regulatory mechanisms, the therapy developing and

Correspondence to: Dr Yandong Yang, Department of Obstetrics and Gynecology, Binzhou Medical University Hospital, 661 Huanghe Second Road, Binzhou, Shandong 256603, P.R. China

E-mail: yangyandong2017@163.com

Key words: preterm, microRNA, targetscore, bioinformatics, variational Bayesian-Gaussian mixture model avoiding adverse effects are difficult. Therefore, making sure the genetic mechanism on the critical aspects of embryonic development is important to predict the occurrence of preterm and ensure smooth delivery (6).

MicroRNA (miRNA) is a small, non-coding, single-stranded RNA, which can participate in the post-transcriptional regulation of gene expression of either mRNA degradation or inhibiting translation, thereby controlling protein synthesis or inhibition (7). miRNAs contain approximately 19-24 nucleotides, and their regulating function on mRNA is accomplished by base-pairing to the 3'-untranslated region (UTR) of target mRNAs (8). However, the same miRNA in the mechanism of different diseases has different target genes and biological functions (9). As early as 1997, scholars found cell-free fetal mRNAs which existed in the blood of pregnant women. The abnormal increase of these cell-free fetal mRNAs was confirmed to have a correlation with preterm (10). Therefore, we believe that the abnormal expression of miRNA-regulated mRNAs have a close contact with the occurrence of preterm. Recently, it was identified that the miRNA-200 family is highly upregulated in myometrium both of mice and humans, and the coordinately downregulated targets are $Z E B 1$ and $Z E B 2$ (11). ZEB1 and ZEB2 could inhibit the contraction-associated gene expression, including the expression of oxytocin receptor and connexin 43. It was believed that if the expression of $Z E B 1$ and $Z E B 2$ is decreased, the transcription of contraction-associated genes leading to delivery is increased. Pineles et al used the real-time quantitative reverse transcription-polymerase chain reaction to compare the different expression of 157 miRNAs in the normal placenta and the premature placenta (12). The differential expression of miRNA-210 and miRNA-182 in the two groups was found, and the specific biology of miRNA-182 also was analyzed. It suggests that miRNA-200 and miRNA-182 may have an important significance for the inchoate diagnosis and prevention of preterm. However, the existing reports, do not entirely predict the mRNAs which are regulated by the relevant miRNAs. In order to clarify the mechanism of preterm and understanding the regulatory role of miRNAs in premature delivery, we selected miRNA-200 and miRNA-182 to predict the target genes.

Because every miRNA has numerous target mRNAs, the accurate prediction on target mRNAs is difficult to characterize. The common solution for this problem is to first predict 
the target mRNAs by the bioinformatics tools, and then to verify these miRNA: mRNA interactions by experiment (13). Presently, the common method is the TargetScan algorithm which predicts mRNAs by the principle of sequence complementarity. In order to improve the accuracy of the prediction, we predict the target genes by the targetscore algorithm which is developed on the basis of TargetScan algorithm. The mathematical model of targetscore algorithm is the variational Bayesian-Gaussian mixture model (VB-GMM) which was modified on the basis of prediction model revised by Nam in 2014 (14). Unlike the TargetScan method, it is not limited to predict conserved genes, so it has a higher accuracy which could improve the efficiency of experimental verification later and reduce the error rate.

In this study, we used the targetscore method to predict the target genes of miRNA-200 and miRNA-182 which affect preterm. Furthermore, we used the $\log \mathrm{FCl}$ and targetscore value of each gene to determine the ultimate prediction results. The target genes of miRNA-200 are KIAA0754, POGZ, ACKR3, N4BP2, RAMP3, GS1-259H13.2, SPX, MAST4, KRBA2, PFN2, RNF145, SYDE2, RIN2, ZNF665, ACOXL, NMRK1, TSNAX, ZNF274, DENND4B, and SGK494. The target genes of miRNA-182 are KIAA1549, AMOTL2, NUMB, NRN1, TARBP1, SAMD5, KLHL5, FAM107A, TSPAN14, DACT2, EP300, RASGRP1, CA10, ATM, OTOGL, KIF3B, SYDE2, MAST4, TSNAX, and LCOR.

\section{Materials and methods}

miRNA expression profile data. We obtained the miRNA expression profile GSE18809 which was stored in the Gene Expression Omnibus (GEO) database (http://www.ncbi.nlm. nih.gov/geo/) by Stephen and other scholars. The GSE18809 dataset based on GPL570 contained 10 samples, including 5 placentas of term and 5 placentas of preterm spontaneous delivery samples. The placentas were all obtained from Prince of Wales Hospital in Hong Kong (China). Each placental biopsy was collected, rinsed in phosphate-buffered saline, and submerged into RNA later (Ambion: Thermo Fisher Scientific, Inc., Waltham, MA, USA) as soon as possible after delivery.

Preprocessing of genes. The miRNA expression profile is RNA. We preprocessed the expression profile data and obtained the relative expression quantity which is more advantageous to follow-up gene differences in the calculation. The relative expression quantity cannot include the missing value, the nearly unvaried value, the maximum value and the minimum value. Therefore, the gene expression we obtained does not contain these values. We selected the database hgu133plus2 for gene preprocessing. The new gene expression set consists of the expression values and the sample annotation information. In the first step of preprocessing, we sorted out the expression values of all genes. If there are several probes which represent the same gene, we calculated the average of these probes as the expression value of this gene. Then, we modified the sample annotation information and renamed the groups as 'preterm' and 'control'. Finally, we reprocessed the gene expression values and changed the row name of the expression matrix to GENESYMBOL.
The calculation of targetscore. We use the targetscore algorithm to predict the target gene for miRNAs. The targetscore method is improved based on the TargetScan method through the algorithms which can improve match prediction accuracy. It passed by genome-wide comparison of differential gene expression to predict the target genes without starting to rely on evolutionary conservation (15). We deduced the maximum likelihood by the VB-GMM, and obtained the posterior probability of each gene. In other words, the posterior probability is calculated by the prior probability and the likelihood function.

First of all, we used the limma package to calculate the logFC value of all genes. The limma package provides a framework for analyzing gene expression experiments from beginning to end in a flexible and statistically rigorous way (16). It is often used to detect differently expressed genes. In this study, we do not need to detect differentially expressed genes, but we had to calculate the $\log \mathrm{FC}$ values of each gene by limma package. Therefore, we calculated the $\log \mathrm{FC}$ values for each gene with the data of the miRNA expression profile. And the calculated $\log \mathrm{FC}$ value is known as the priori value in target gene prediction. The next step is to calculate the posterior value with the equation. This algorithm is available at www.genelibs.com/gb. TargetScan context score (TSCS) and the probabilities of conserved targeting (PCT) data of the desired gene are acquired in the TargetScan data website. Based on the TSCS value, the PCT value and the experimental $\log \mathrm{FC}$ value, we inferred the maximum likelihood value by the VB-GMM. In other words, the posterior value of each gene is calculated with the prior probability and likelihood function. The variational Bayesian algorithm is a process of iterating to optimize the maximum likelihood estimate. It is based on variational principle to find the edge distribution of minimality Kullback-Leibler distance to approximate the joint distribution, and use the mean field to reduce the complexity of the joint estimation. It is the reason that we regarded the targetscore method as an extension of the Expectation Maximization (EM) algorithm in some ways. The VB-GMM model follows the standard Bayesian GMM reported by Bishop with only tiny modifications. We initialized the model parameters based on priors and randomly sample $\kappa$ data points $\mu$, then we evaluated the Equation 1 using the model parameters and update the model parameters using Equation 1 at the ith iteration.

$$
\mathrm{p}(\mathrm{Znk} \mid \chi \mathrm{n}, \theta) \equiv \sum[\mathrm{Znk}]=\frac{\rho \mathrm{nk}}{\sum_{\mathrm{j}=1}^{\mathrm{k}} \rho \mathrm{nj}}
$$

Finally, we calculated the targetscore value and predicted the target gene for the miRNA according to the targetscore value of each gene. The targetscore is defined as an integrative probabilistic score of a target gene of a miRNA. The targetscore value is calculated by the Equation 2, and the target genes of chosen miRNAs are evaluated quantitatively according to the value.

$$
\text { Targetscore }=\frac{1}{1+\mathrm{e}^{x}} \times \bar{p}
$$

In Equation 2, the $\mathrm{x}$ means the $\log \mathrm{FC}$ value of the genes which calculate by limma package, and the $\mathrm{p}$ means the posterior value inferred. 


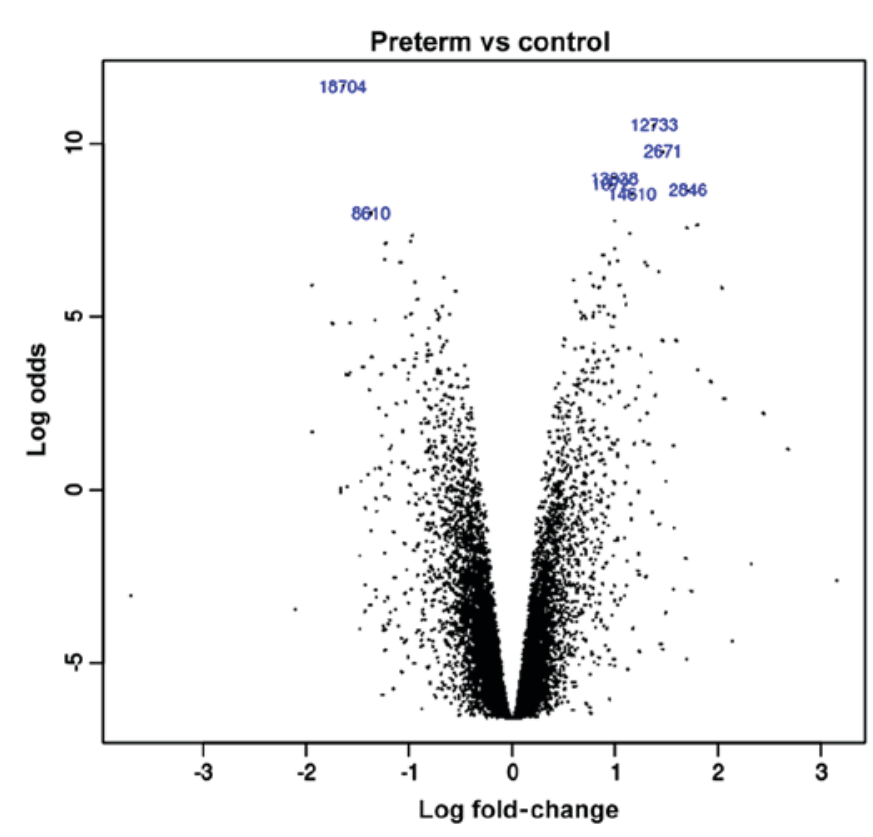

Figure 1. The $\log \mathrm{FCl}$ of all genes. Each black spot represents a gene in the volcano plot of the $\log \mathrm{FC}$. The result in the chart is the $\log \mathrm{FC}$ value of the gene obtained by the limma package. Compared with the normal placenta, there are 9,783 upregulated genes and 10,731 downregulated genes in preterm placenta.

\section{Results}

The volcano plot of the $\log F C$. In this study, we extracted RNA from 5 cases of preterm and 5 cases of normal placenta for analysis. Through the mapping between the probe and the gene we obtained a total of 20,514 genes. We used the limma package to calculate the discrepant expression of the genes between the preterm placenta experimental group and the normal placenta control group. Then we obtained the logFC value of each gene according to the data with linear fit. The acquired $\log \mathrm{FC}$ value is regarded as a priori value in our study and all data are integrated into the volcano plot (Fig. 1). In all different genes, there are 9,783 downregulated genes and 10,731 upregulated genes.

Preliminary screening of the target genes with the targetscore. We predicted the target genes of the chosen miRNAs, and the targetscore $=0.62$ was considered as the preliminary screening level. After preliminary screening, we selected 140 target genes of miRNA-200 and 260 target genes of miRNA-182. The target genes of miRNA-200 are all non-validated, while the CLOCK, EP3OO, FOXO1, and FOXO3 are validated target genes of miRNA-182. In order to increase the accuracy of target gene prediction, we made further target gene screening with the higher targetscore values and drew the top 50 target genes into the cytoscape chart (Fig. 2).

Re-screening of the target genes. In order to make the results more accurate, we re-screened the predicted target genes. We used the $\log \mathrm{FCl}$ and targetscore value as the quantitative standard of re-screening. In all $\log \mathrm{FCl}$ value $>0.6$ of predicted genes, we selected 20 target genes which were the most connected with miRNAs as the final screening results.

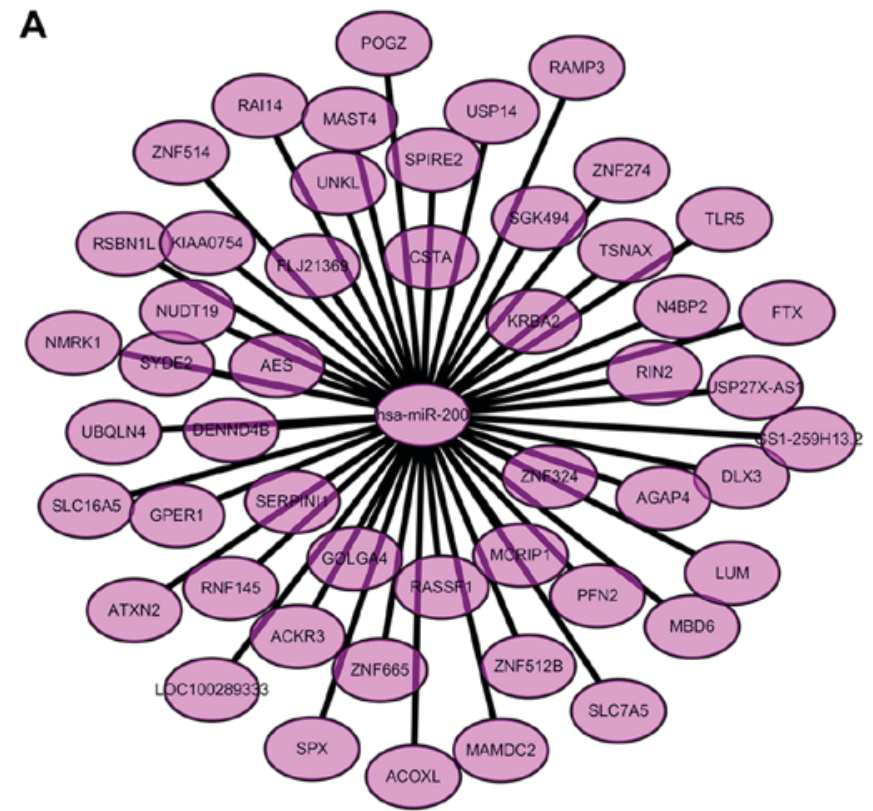

The top 50 target genes of miRNA-200

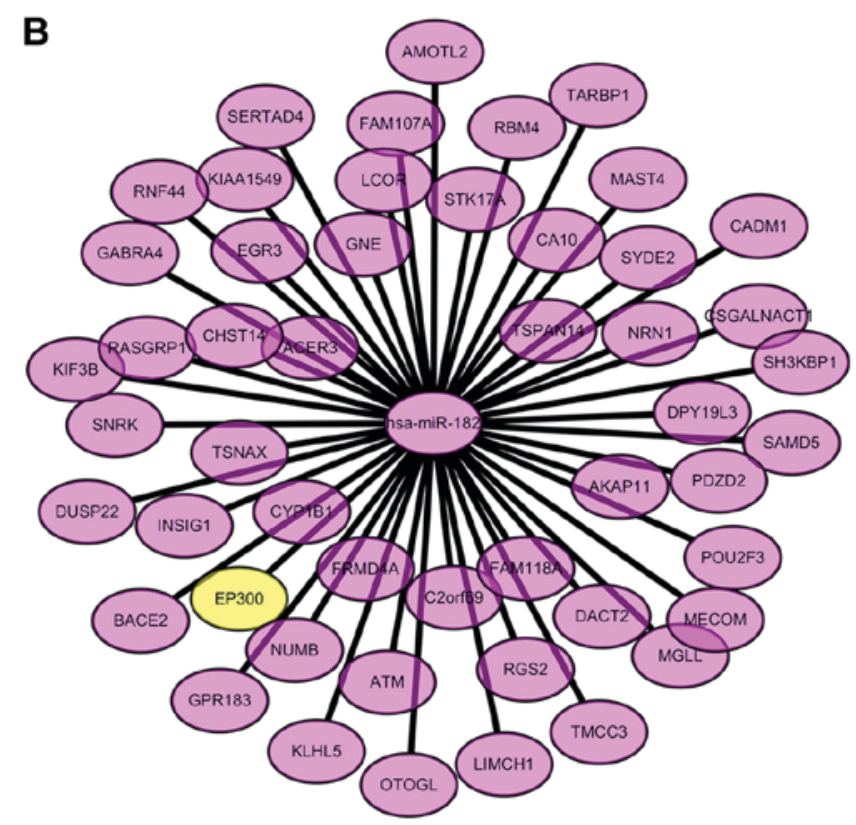

The top 50 target genes of miRNA-182

Figure 2. The prediction of target genes use targetscore algorithm and the results are preliminary screening. The predicted target genes of (A) miRNA-200, and (B) miRNA-182. The values between the miRNAs and the predicted genes are the targetscore and the yellow-labeled genes are the validated target genes. miRNA, microRNA.

Since the targetscore value is calculated by TargetScan CS, TargetScan PCT, and logFC value, the relevance between the target gene and the miRNA is measured by the targetscore value. Thus, we re-screened the predictions of miRNA-200 and miRNA-182 by the $\log \mathrm{FCl}$ values and targetscore values. The target genes of miRNA-200 are KIAA0754, POGZ, ACKR3, N4BP2, RAMP3, GS1-259H13.2, SPX, MAST4, KRBA2, PFN2, RNF145, SYDE2, RIN2, ZNF665, ACOXL, NMRK1, TSNAX, ZNF274, DENND4B, and SGK494. The target genes of miRNA-182 are KIAA1549, AMOTL2, NUMB, 
Table I. The miRNA-200 and miRNA-182 target genes.

\begin{tabular}{|c|c|c|c|c|c|}
\hline \multicolumn{3}{|c|}{ miRNA-200 } & \multicolumn{3}{|c|}{ miRNA-182 } \\
\hline External gene ID & $\operatorname{LogFC}$ & Targetscore & External gene ID & $\operatorname{LogFC}$ & Targetscore \\
\hline KIAA0754 & -0.65328 & 0.622305 & KIAA1549 & -0.800179644 & 0.657947267 \\
\hline POGZ & -0.65319 & 0.622305 & AMOTL2 & -0.774416547 & 0.657750851 \\
\hline ACKR3 & -0.65379 & 0.622305 & $N U M B$ & -0.810013576 & 0.657727 \\
\hline$N 4 B P 2$ & -0.65401 & 0.622305 & $N R N 1$ & -0.770132168 & 0.657618084 \\
\hline$R A M P 3$ & -0.6541 & 0.622305 & TARBPI & -0.767998978 & 0.657542041 \\
\hline GS1-259H13.2 & -0.65426 & 0.622305 & SAMD5 & -0.744871627 & 0.656319805 \\
\hline$S P X$ & -0.65236 & 0.622304 & KLHL5 & -0.839652128 & 0.655947693 \\
\hline MAST4 & -0.65219 & 0.622304 & FAM107A & -0.848040295 & 0.655109486 \\
\hline KRBA2 & -0.65135 & 0.622302 & TSPAN14 & -0.728294334 & 0.655035392 \\
\hline PFN2 & -0.65558 & 0.622301 & $D A C T 2$ & -0.725113964 & 0.654753677 \\
\hline RNF145 & -0.65111 & 0.622301 & EP300 & -0.720548552 & 0.654330512 \\
\hline SYDE2 & -0.65576 & 0.6223 & $R A S G R P 1$ & -0.855821334 & 0.654202657 \\
\hline RIN2 & -0.65084 & 0.6223 & CA10 & -0.863356689 & 0.65318794 \\
\hline ZNF665 & -0.65602 & 0.622299 & ATM & -0.705431839 & 0.652779173 \\
\hline$A C O X L$ & -0.65035 & 0.622297 & OTOGL & -0.697877318 & 0.65192387 \\
\hline NMRKI & -0.6501 & 0.622296 & KIF $3 B$ & -0.6717802 & 0.648598376 \\
\hline TSNAX & -0.64971 & 0.622293 & SYDE2 & -0.655760282 & 0.646305057 \\
\hline ZNF274 & -0.6495 & 0.622292 & MAST4 & -0.652193502 & 0.645771108 \\
\hline DENND4B & -0.649 & 0.622288 & TSNAX & -0.649707123 & 0.645394125 \\
\hline SGK494 & -0.64895 & 0.622288 & $L C O R$ & -0.635573098 & 0.643180143 \\
\hline
\end{tabular}

miRNA, microRNA.

Table II. The reference information of validated gene.

\begin{tabular}{lcccc}
\hline $\begin{array}{l}\text { Target } \\
\text { gene }\end{array}$ & $\begin{array}{c}\text { Target gene } \\
\text { (Entrez ID) }\end{array}$ & Experiments & $\begin{array}{c}\text { Support } \\
\text { type }\end{array}$ & $\begin{array}{c}\text { References } \\
\text { (PMID) }\end{array}$ \\
\hline EP300 & 2033 & Microarray & $\begin{array}{c}\text { Functional } \\
\text { MTI (weak) }\end{array}$ & 19569050 \\
\hline
\end{tabular}

NRN1, TARBP1, SAMD5, KLHL5, FAM107A, TSPAN14, DACT2, EP300, RASGRP1, CA10, ATM, OTOGL, KIF3B, SYDE2, MAST4, TSNAX, and LCOR (Table I). In these genes, only EP300 is a validated target gene, so we reorganized the reference information into a table (Table II).

\section{Discussion}

miRNAs have important functions because the post-transcriptional level regulation is accomplished by them binding to the 3'-UTR of mRNAs. Some studies have shown that many miRNAs are dysregulated in preterm placentas, and the occurrence of preterm has significant correlations with the different expression levels of these miRNAs (17). The disordered miRNAs include miRNA-200 and miRNA-182. The relationship between miRNAs and their target genes is dynamic in different conditions. Therefore, the functions of miRNAs are different in each context. For the occurrence of preterm, it is considered that miRNAs have an effect on gene regulation of uterine quiescence/contractility during pregnancy and delivery (18). Based on these studies, Williams et al confirmed that miRNA-200, which is highly upregulated in myometrium of both human and mouse, is an important factor to control progesterone (P4) and progesterone receptor (PR) expression (11). Furthermore, the induction of miR-200 family expression led to the decline of PR function and the increase of contraction-associated gene transcription. For this reason, the occurrence of preterm is closely associated with the abnormal expression of miRNA-200. The scholars have found that some specific miRNAs were abnormally upregulated in placentas of preeclampsia and small-for-gestational age neonates, including miRNA-182. The relevance between preeclampsia and upregulated miRNA-182 expression indicates the possibility of a functional role for miRNA-182 in preeclampsia or preterm (12). However, these studies did not disclose the exact target genes which are regulated by miRNA-200 and miRNA-182, so that it cannot completely clarify the mechanism of preterm. The potential target gene identification is fundamental for the explanation of the biological role of individual miRNAs in vivo. Therefore, we used the targetscore algorithm to reveal the target genes of miRNAs in the preterm to provide evidence for variations in miRNA expression in obstetric disease.

As the accurate prediction of target mRNAs is difficult to characterize, an increasing number of new bioinformatics tools have been developed to improve the accuracy of prediction. 
TargetScan is the first algorithm used to predict miRNA vertebrate targets. The first edition of TargetScan algorithm was developed in 2003 by Bartel's group (19). In order to improve match prediction accuracy scholars utilized more databases and genomes to develop new versions of this algorithm, such as TargetScanS, TargetScan PCT, TSCS (20-22). In our study, we chose the targetscore algorithm. This algorithm calculates the score for each of the context first and then weights the score by the AIR of a specific site in each cell type, and obtains the cell-type-specific score for each site ultimately. Compared with other versions of the TargetScan algorithms, the targetscore algorithm is more closely related to preterm, and the predicted target genes of miRNA are more specific.

miRNAs achieve their biological function by the regulation of downstream target genes, and it is estimated that $>30 \%$ of human genes are potential targets for miRNAs. In order to ascertain the mechanism of action of miRNAs in preterm, it is crucial to predict their target genes. By predicting the target genes, it is helpful to understand the expression patterns in placenta of distinct pathologies and provide the theoretical basis for the prevention and drug development of preterm. We calculated the targetscore values with the TSCS, PCT and $\operatorname{logFC}$ values. For this purpose, the prediction results are closer to the disease and more accurate. After prediction, we found that there were 9,783 downregulated genes and 10,731 upregulated genes in the differentially expressed genes. At targetscore $=0.62$, there are 140 miRNA-200 target genes and 260 miRNA-182 target genes were selected. In order to further confirm the predicted results accurately, we used $\log \mathrm{FCl}$ and targetscore values to select the target genes which are more closely related to miRNA in occurrence of preterm. The prediction results of miRNA-200 are KIAA0754, POGZ, ACKR3, N4BP2, RAMP3, GS1-259H13.2, SPX, MAST4, KRBA2, PFN2, RNF145, SYDE2, RIN2, ZNF665, ACOXL, NMRK1, TSNAX, ZNF274, $D E N N D 4 B$, and $S G K 494$. The target genes of miRNA-182 are KIAA1549, AMOTL2, NUMB, NRN1, TARBP1, SAMD5, KLHL5, FAM107A, TSPAN14, DACT2, EP300, RASGRP1, CA10, ATM, OTOGL, KIF3B, SYDE2, MAST4, TSNAX, and $L C O R$. However, in all results, EP300 is the only gene which is validated by the other scholars (23). It means that the study of preterm birth mechanism is still a great shortage, and a lot of genes are still not experimentally verified. This suggests that in the future study of mechanism we can regard the other non-validated target genes as the research goal and to explore the influence of miRNA-200 and miRNA-182 on the preterm control though their differential expression. We carefully read the literature which described the only validated target gene EP300. Mees et al analyzed the expression of the mRNAs which were regulated by the specific expressed miRNAs during development and metastasis of pancreatic ductal adenocarcinoma (23). By comparing the gene expression profiles of the biological groups with different metastatic potential, they identified EP300 as the most important target mRNA of miRNA-182 and other significantly upregulated miRNAs in an alignment of the epigenetic and genetic expression profiling. This conclusion to some extent shows that the predicted target genes in our study are credible.

The targetscore algorithm model which is specifically designed for miRNA-overexpression experiments to interrogate targets of a particular miRNA in a specific cell condition is different from the overexpression model of miRNA coupled with expression profiling of mRNA by either microarray or RNA-seq. In our study, we respectively selected 20 genes as the target genes which are regulated by miRNA-200 and miRNA-182 to be affected preterm based on the $\mid \operatorname{logFCl}$ and targetscore values of each gene. In the results, EP300 is one of the validated target genes regulated by miRNA-182. This suggests that our predictions are credible. It can effectively control the functional validation of prediction genes and regulate the drug design in the future, while it will have an important significance to prevent the preterm early.

\section{Acknowledgements}

Not applicable.

\section{Funding}

No funding was received.

\section{Availability of data and materials}

The datasets used and/or analyzed during the current study are available from the corresponding author on reasonable request.

\section{Authors' contributions}

KL performed the experiments and analyzed the data, and was also a major contributor in writing the manuscript. JH and YY made a substantial contribution to the conception and design of the study. KL, JH and YY performed the statistical analysis. DL was involved in the conception and design of the study. All authors read and approved the manuscript.

\section{Ethics approval and consent to participate}

Not applicable.

\section{Patient consent for publication}

Not applicable.

\section{Competing interests}

The authors declare that they have no competing interests.

\section{References}

1. Gray C, McCowan LM, Patel R, Taylor RS and Vickers MH: Maternal plasma miRNAs as biomarkers during mid-pregnancy to predict later spontaneous preterm birth: A pilot study. Sci Rep 7: 815, 2017

2. Peixoto AB, da Cunha Caldas TMR, Tahan LA, Petrini CG, Martins WP, Costa FDS and Araujo Júnior E: Second trimester cervical length measurement for prediction spontaneous preterm birth in an unselected risk population. Obstet Gynecol Sci 60: 329-335, 2017.

3. Kalikstad B, Kultima HG, Andersstuen TK, Klungland A and Isaksson A: Gene expression profiles in preterm infants on continuous long-term oxygen therapy suggest reduced oxidative stress-dependent signaling during hypoxia. Mol Med Rep 15: 1513-1526, 2017.

4. Chen Y, Song Y, Huang J, Qu M, Zhang Y, Geng J, Zhang Z, Liu J and Yang GY: Increased circulating exosomal miRNA-223 is associated with acute ischemic stroke. Front Neurol 8: 57, 2017. 
5. Dutta S, Singh S, Bhattacharya A, Venkataseshan S and Kumar P: Relation of thyroid hormone levels with fluid-resistant shock among preterm septicemic neonates. Indian Pediatr 54: 121-124, 2017

6. Bukowski R, Sadovsky Y, Goodarzi H, Zhang H, Biggio JR, Varner M, Parry S, Xiao F, Esplin SM, Andrews W, et al: Onset of human preterm and term birth is related to unique inflammatory transcriptome profiles at the maternal fetal interface. PeerJ 5: e3685, 2017.

7. Anton L, DeVine A, Sierra LJ, Brown AG and Elovitz MA: miR-143 and miR-145 disrupt the cervical epithelial barrier through dysregulation of cell adhesion, apoptosis and proliferation. Sci Rep 7: 3020, 2017

8. Sanders AP, Burris HH, Just AC, Motta V, Svensson K, Mercado-Garcia A, Pantic I, Schwartz J, Tellez-Rojo MM, Wright RO, et al: microRNA expression in the cervix during pregnancy is associated with length of gestation. Epigenetics 10 221-228, 2015.

9. Brunquell J, Snyder A, Cheng F and Westerheide SD: HSF-1 is a regulator of miRNA expression in Caenorhabditis elegans. PLoS One 12: e0183445, 2017

10. Mayor-Lynn K, Toloubeydokhti T, Cruz AC and Chegini N: Expression profile of microRNAs and mRNAs in human placentas from pregnancies complicated by preeclampsia and preterm labor. Reprod Sci 18: 46-56, 2011.

11. Williams KC, Renthal NE, Condon JC, Gerard RD and Mendelson CR: MicroRNA-200a serves a key role in the decline of progesterone receptor function leading to term and preterm labor. Proc Natl Acad Sci USA 109: 7529-7534, 2012

12. Pineles BL, Romero R, Montenegro D, Tarca AL, Han YM, Kim YM, Draghici S, Espinoza J, Kusanovic JP, Mittal P, et al: Distinct subsets of microRNAs are expressed differentially in the human placentas of patients with preeclampsia. Am J Obstet Gynecol 196: 261.e1-261.e6, 2007.

13. L'Yi S, Jung D, Oh M, Kim B, Freishtat RJ, Giri M, Hoffman E and Seo J: miRTarVis+: Web-based interactive visual analytics tool for microRNA target predictions. Methods 124: 78-88, 2017.

14. Nam JW, Rissland O, Koppstein D, Abreu-Goodger C, Jan CH, Agarwal V, Yildirim MA, Rodriguez A and Bartel DP: Global analyses of the effect of different cellular contexts on microRNA targeting. Molecular Cell 53: 1031-1043, 2014.
15. Li Y, Goldenberg A, Wong KC and Zhang Z: A probabilistic approach to explore human miRNA targetome by integrating miRNA-overexpression data and sequence information. Bioinformatics 30: 621-628, 2014.

16. Ritchie ME, Phipson B, Wu D, Hu Y, Law CW, Shi W and Smyth GK: limma powers differential expression analyses for RNA-sequencing and microarray studies. Nucleic Acids Res 43: e47, 2015

17. Winger EE, Reed JL and Ji X: Early first trimester peripheral blood cell microRNA predicts risk of preterm delivery in pregnant women: Proof of concept. PLoS One 12: e0180124, 2017.

18. Zhu Q, Chen Y, Dai J, Wang B, Liu M, Wang Y, Tao J and Li H: Methylenetetrahydrofolate reductase polymorphisms at 3'-untranslated region are associated with susceptibility to preterm birth. Transl Pediatr 4: 57-62, 2015.

19. Lewis BP, Shih IH, Jones-Rhoades MW, Bartel DP and Burge CB: Prediction of mammalian microRNA targets. Cell 115: 787-798, 2003

20. Salehi $\mathrm{Z}$ and Akrami H: Target genes prediction and functional analysis of microRNAs differentially expressed in gastric cancer stem cells MKN-45. J Cancer Res Ther 13: 477-483, 2017.

21. Zhao B and Xue B: Consensus datasets of mouse miRNA-mRNA interactions from multiple online resources. Data Brief 14: 143-147, 2017.

22. Shi Y, Yang F, Wei S and Xu G: Identification of key genes affecting results of hyperthermia in osteosarcoma based on integrative ChIP-Seq/TargetScan analysis. Med Sci Monit 23: 2042-2048, 2017

23. Mees ST, Mardin WA, Wendel C, Baeumer N, Willscher E, Senninger N, Schleicher C, Colombo-Benkmann M and Haier J: EP300 - a miRNA-regulated metastasis suppressor gene in ductal adenocarcinomas of the pancreas. Int J Cancer 126: 114-124, 2010.

This work is licensed under a Creative Commons Attribution-NonCommercial-NoDerivatives 4.0 International (CC BY-NC-ND 4.0) License. 